\title{
An approximate method for the acoustic attenuating orthorhombic eikonal equation
}

\author{
Qi Hao*; Tariq Alkhalifah, KAUST
}

\section{SUMMARY}

Solving the eikonal equation is used widely in traveltime calculation, tomography, Kirchhoff migration etc. The complex eikonal equation governs the traveltimes in an attenuating medium, where the real and imaginary parts of the traveltimes are associated with the phase and energy-absorption, respectively. Attenuating orthorhombic anisotropy can be used to explain the azimuthal variation of velocity- and attenuationanisotropy measured from surface seismic data. We present an approximate method to solve the acoustic eikonal equation for an attenuating orthorhombic medium. We combine perturbation theory and Shanks transform in different ways to derive the analytic solutions in the case of homogeneous media. We design a fast marching scheme to solve the acoustic eikonal equation numerically. We share some numerical examples to demonstrate the effectiveness of the complex eikonal equation in predicting attenuation.

\section{INTRODUCTION}

In an attenuating medium, traveltimes of body-waves are complex valued and are governed by the complex-valued eikonal equation. The real part of the complex valued traveltimes corresponds to the phase of waves, while the imaginary part admits a decay in the amplitude of waves due to energy absorption. The complex traveltimes can be used in inversion for interval attenuation-parameters, attenuation tomography and Kirchhoff imaging with absorption compensation. For attenuating transversely isotropic and orthorhombic media, Hao and Alkhalifah (2017a,b) proposed the acoustic attenuating eikonal equations. For an attenuating transversely isotropic medium with a vertical symmetry axis, they combined perturbation theory and Shanks transform to solve the acoustic eikonal equation and concluded that the parameterization involving the horizontal velocity leads to more accurate results than that involving the NMO velocity (Hao and Alkhalifah, 2017c). Since all of the symmetry planes of an orthorhombic medium exhibit transversely isotropic, it is believable that the conclusion is still valid for orthorhombic media.

In this expanded abstract, we develop an approximate method to solve the acoustic eikonal equation (Hao and Alkhalifah, 2017a) for attenuating orthorhombic media. The modified Alkhalifah's (2000) notation and Zhu and Tsvankin's (2007) notation are combined to describe the P-wave traveltimes in an attenuating orthorhombic medium, where the modified Alkhalifah's notation and Zhu and Tsvankin's notation correspond to the nonattenuating and attenuating parts of the orthorhombic medium, respectively. Similar to the approach used by Hao and Alkhalifah (2017c), perturbation theory and Shanks transform are combined to derive the governing equations for the traveltime expansion and improve the accuracy of traveltime approximation. We solve the governing equations analytically for the case of homogeneous media. We design a fast marching scheme to solve the governing equations in the case of inhomogeneous media.

\section{THE ATTENUATING EIKONAL EQUATION}

The acoustic eikonal equation for an attenuating orthorhombic medium is given by (Hao and Alkhalifah, 2017a)

$$
\operatorname{det}\left(\begin{array}{ccc}
a_{11} \tau_{, x}^{2}-1 & a_{12} \tau_{, x} \tau_{, y} & a_{13} \tau_{, x} \tau_{, z} \\
a_{12} \tau_{, x} \tau_{, y} & a_{22} \tau_{, y}^{2}-1 & a_{23} \tau_{, y} \tau_{, z} \\
a_{13} \tau_{, x} \tau_{, z} & a_{23} \tau_{, y} \tau, z & a_{33} \tau_{, z}^{2}-1
\end{array}\right)=0
$$

where $\tau_{, x}, \tau_{, y}$ and $\tau_{, z}$ denote the partial derivatives of the complex traveltime $\tau$ with respect to $x, y$ and $z$, and $a_{i j}$ are expressed with

$$
\begin{aligned}
& a_{11}=v_{x}^{2}\left(1-2 i k\left(1+\varepsilon_{Q 2}\right)\right), \\
& a_{12}=\frac{v_{x} v_{y}}{r_{3}}\left(1-2 i k\left(1+\varepsilon_{Q 2}\right)\right)-i k \delta_{Q 3}\left(1+\varepsilon_{Q 2}\right) \frac{v_{P 0}^{3}}{v_{y} r_{3}}, \\
& a_{13}=\frac{v_{P 0} v_{x}}{r_{2}}(1-2 i k)-i k \delta_{Q 2} r_{2} \frac{v_{P 0}^{3}}{v_{x}} \\
& a_{22}=v_{y}^{2}\left(1-2 i k\left(1+\varepsilon_{Q 1}\right)\right), \\
& a_{23}=\frac{v_{P 0} v_{y}}{r_{1}}(1-2 i k)-i k \delta_{Q 1} r_{1} \frac{v_{P 0}^{3}}{v_{y}} \\
& a_{33}=v_{P 0}^{2}(1-2 i k),
\end{aligned}
$$

with

$$
\begin{gathered}
k_{Q}=\frac{A_{P 0}}{1-A_{P 0}^{2}}, \\
r_{1}=\sqrt{1+2 \eta_{1}}, \quad r_{2}=\sqrt{1+2 \eta_{2}}, \quad r_{3}=\sqrt{1+2 \eta_{3}},
\end{gathered}
$$

where $i$ denotes the imaginary unit, $v_{P 0}$ denotes the P-wave vertical velocity in the nonattenuating reference medium, $v_{x}$ and $v_{y}$ denotes the $\mathrm{P}$-wave horizontal velocities along the $x$ and $y$-axes in the reference medium, $\eta_{1}, \eta_{2}$ and $\eta_{3}$ denote the anellipticity parameters defined in the $[y, z],[x, z]$ and $[x, y]$ planes, $A_{P 0}$ denotes the wavenumber-normalized attenuation coefficient of the vertically propagating P-wave, $\varepsilon_{Q 1}$ and $\delta_{Q 1}$ are the attenuation anisotropy parameters defined in the $[y, z]$ plane, $\varepsilon_{Q 2}$ and $\delta_{Q 2}$ are the attenuation anisotropy parameters defined in the $[x, z]$ plane, and $\delta_{Q 3}$ are the attenuation anisotropy parameters defined in the $[x, y]$ plane.

\section{THE GOVERNING EQUATIONS}

The eikonal equation 2 is generally expressed in their hamiltonian form as

$$
F\left(\tau, \eta_{1}, \eta_{2}, \eta_{3}, i k_{Q}\right)=0,
$$

where $F$ represents the left side of the eikonal equation, the product of $i$ and $k_{Q}$ is taken as the fourth parameter, since $i$ always appears together with $k_{Q}$ in the eikonal equation. 


\section{Complex traveltimes in attenuating orthorhombic media}

To seek an approximate solution, we introduce the perturbation parameters $\zeta_{i}$ to scale $\eta_{i}$ and $k_{Q}$ in the eikonal equation,

$$
F\left(\tau, \zeta_{1} \eta_{1}, \zeta_{2} \eta_{2}, \zeta_{3} \eta_{3}, i \zeta_{4} k_{Q}\right)=0 .
$$

The second-order trial solution to the above equation is defined as

$$
\tau=\tau_{0}+\sum_{i=1}^{4} \tau_{i} \tilde{\zeta}_{i}+\sum_{i \leq j=1}^{4} \tau_{i j} \tilde{\zeta}_{i} \tilde{\zeta}_{j}
$$

with

$$
\tilde{\zeta}=\left(\zeta_{1}, \zeta_{2}, \zeta_{3}, i \zeta_{4}\right)^{T}
$$

where $\tau_{0}, \tau_{i}$ and $\tau_{i j}$ denote the zeroth-, first- and second-order traveltime coefficients, respectively. Substituting the trial solution 7 into equation 6 and then expanding it with respect to the perturbation parameters up to second-order, we derive the governing equations for the traveltime coefficients as follows.

- The governing equation for $\tau_{0}$ :

$$
v_{x}^{2} \tau_{0, x}^{2}+v_{y}^{2} \tau_{0, y}^{2}+v_{P 0}^{2} \tau_{0, z}^{2}=1 .
$$

- The governing equation for $\tau_{i}(i=1,2,3)$ :

$$
v_{x}^{2} \tau_{0, x} \tau_{i, x}+v_{y}^{2} \tau_{0, y} \tau_{i, y}+v_{P 0}^{2} \tau_{0, z} \tau_{i, z}=f_{i}\left(\tau_{0}\right)
$$

- The governing equation for $\tau_{i j}(i \leq j=1,2,3)$ :

$$
v_{x}^{2} \tau_{0, x} \tau_{i j, x}+v_{y}^{2} \tau_{0, y} \tau_{i j, y}+v_{P 0}^{2} \tau_{0, z} \tau_{i j, z}=f_{i j}\left(\tau_{0}, \tau_{i}, \tau_{j}\right) .
$$

All of the traveltime coefficients can be obtained by solving the governing equations successively. For a homogeneous attenuating orthorhombic medium, all of the governing equations are analytically solvable.

\section{THE SERIES AND SHANKS SOLUTIONS}

Let us assume that the traveltime coefficients $\tau_{0}, \tau_{i}$ and $\tau_{i j}$ have been calculated using equations 9-11. The second-order series solution for the traveltimes is obtained from equation 7 by setting all the perturbation coefficients $\zeta_{i}=1$. Applying Shanks transform to equation 7 in different ways and then setting $\zeta_{i}=1$, we end up with three possible Shanks solutions. All of these approximate solutions are summarized as follows.

- The second-order series solution:

$$
\tau=\tau_{0}+\sum_{i=1}^{4} \tau_{i} \chi_{i}+\sum_{i \leq j=1}^{4} \tau_{i j} \chi_{i} \chi_{j}
$$

with

$$
\boldsymbol{\chi}=(1,1,1, i)^{T} .
$$

- Shanks transform solution 1:

$$
\tau=T_{0}^{(1)}+\frac{\left(T_{1}^{(1)}\right)^{2}}{T_{1}^{(1)}-T_{2}^{(1)}},
$$

with

$$
T_{0}^{(1)}=\tau_{0}, \quad T_{1}^{(1)}=\sum_{i=1}^{4} \tau_{i} \chi_{i}, \quad T_{2}^{(1)}=\sum_{i \leq j=1}^{4} \tau_{i j} \chi_{i} \chi_{j} .
$$

- Shanks transform solution 2:

$$
\tau=T_{0}^{(2)}+\frac{\left(T_{1}^{(2)}\right)^{2}}{T_{1}^{(2)}-T_{2}^{(2)}}
$$

with

$$
T_{0}^{(2)}=\tau_{0}-\tau_{44}+i \tau_{4}, \quad T_{1}^{(2)}=\sum_{i=1}^{3} \tau_{i}+i \sum_{i=1}^{3} \tau_{i 4}, \quad T_{2}^{(2)}=\sum_{i \leq j=1}^{3} \tau_{i j} .
$$

- Shanks transform solution 3:

$$
\tau=T_{0}^{(3)}+\frac{\left(T_{1}^{(3)}\right)^{2}}{T_{1}^{(3)}-T_{2}^{(3)}}
$$

with

$$
T_{0}^{(3)}=\sum_{i=1}^{3} \tau_{i}+\sum_{i \leq j=1}^{3} \tau_{i j}, \quad T_{1}^{(3)}=i \sum_{i=1}^{3} \tau_{i 4}, \quad T_{2}^{(3)}=-\tau_{44} .
$$

Shanks transform solutions 1-3 are formulated with respect to $\zeta_{1}-\zeta_{4}, \zeta_{1}-\zeta_{3}$, and $\zeta_{4}$, respectively.

\section{FAST MARCHING SCHEME}

For an inhomogeneous attenuating orthorhombic medium, we design a fast marching scheme to calculate the traveltimes. The numerical procedure of the scheme is summarized as follows.

First, we calculate the zeroth-order traveltime coefficient $\tau_{0}$ from equation 9 . We adopt the 3D fast marching method, similar to the 2D VTI version used by Alkhalifah (2011), to solve equation 9 .

Next, we calculate the first- and second-order traveltime coefficients $\tau_{i}$ and $\tau_{i j}$ by successively solving equations 10 and 11 in the order of calculating $\tau_{0}$ in the fast marching method.

Last, we calculate the traveltime $\tau$ using Shanks transform solution 2 (equation 16). Here, we picked Shanks transform solution 2 because, as we will see, Shanks transform solution 2 is the most accurate among the proposed solutions.

\section{NUMERICAL EXAMPLES}

In the first example, we would like to compare the accuracy of the series and Shanks transform solutions (equations 12, 14, 16 and 18). We design a homogeneous orthorhombic model with strong attenuation anisotropy. The model parameters include $v_{P 0}=3.0 \mathrm{~km} / \mathrm{s}, v_{x}=3.795 \mathrm{~km} / \mathrm{s}, v_{y}=3.550 \mathrm{~km} / \mathrm{s}, \eta_{1}=0.278$, $\eta_{2}=0.167, \eta_{3}=0.229, A_{P 0}=0.02498$ (corresponding to $\left.Q_{33}=20\right), \varepsilon_{Q 1}=0.66, \delta_{Q 1}=0.52, \varepsilon_{Q 2}=-0.33, \delta_{Q 2}=0.98$, $\delta_{Q 3}=0.94$. The governing equations $9-11$ are solved analytically to obtain the traveltime coefficients. The detailed procedure to calculate the exact traveltimes is shown in Vavryčuk $(2006,2007)$. For the real part of the traveltimes, as shown in 


\section{Complex traveltimes in attenuating orthorhombic media}

Figures 1-3, Shanks transform solutions 1 and 2 (equations 14 and 16) are more accurate than the series solution (equation 12) and Shanks transform solution 2 (equation 18). For the imaginary part of the traveltimes, as shown in Figures 4-6, Shanks transform solution 2 is the most accurate among the proposed solutions. A comprehensive comparison between Figures 1-6 indicates that Shanks transform solution 2 is the most accurate for both the real and imaginary parts of the traveltimes.

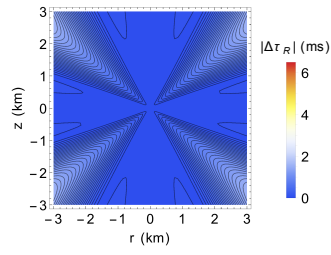

(a)

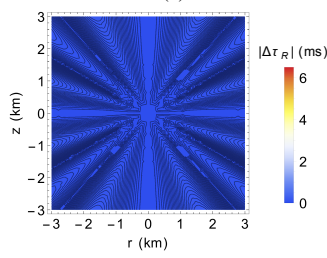

(c)

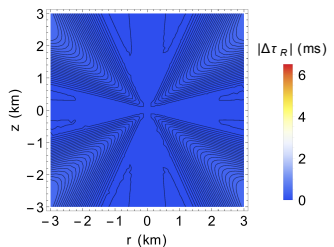

(b)

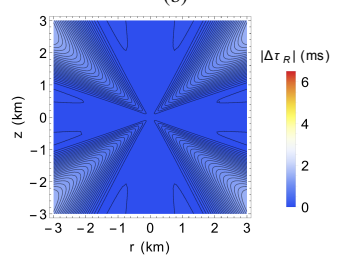

(d)
Figure 1: Errors in the real part of the traveltimes, along the observation azimuth of $0^{\circ}$, from (a) the series solution (equation 12), (b) the Shanks solution 1 (equation 14), (c) the Shanks solution 2 (equation 16) and (d) the Shanks solution 3 (equation 18), respectively. The observation azimuth is measured from the $x$-direction in the horizontal plane. The source is located at the origin of the coordinate system. $r$ denotes the radial coordinate.

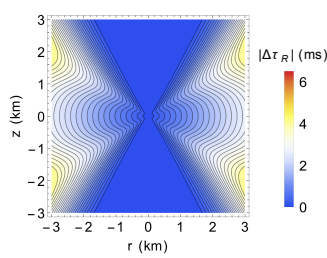

(a)

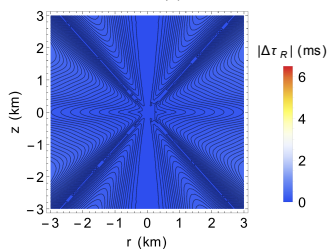

(c)

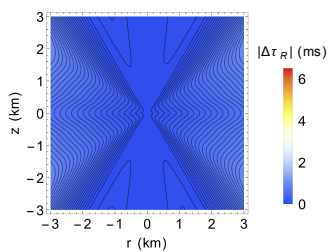

(b)

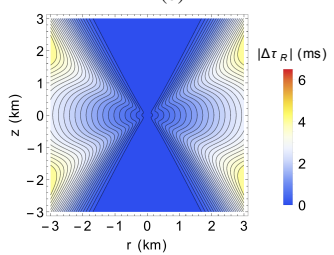

(d)
Figure 2: Similar to Figure 1, but along the azimuth of $45^{\circ}$.

In the second example, we would test the fast marching scheme. We adopt the same attenuating orthorhombic model as shown in the first example. Figure 7 shows a comparison between the accurate analytic traveltimes from the Shanks solution 2 (equation 16) and the numerical traveltimes from the fast marching scheme. Both the real and imaginary parts of the analytic traveltimes match well with those of the numerical traveltimes.

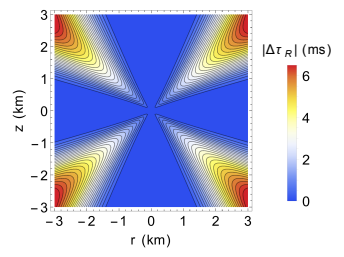

(a)

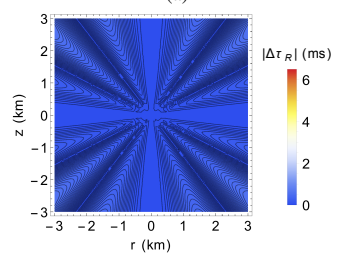

(c)

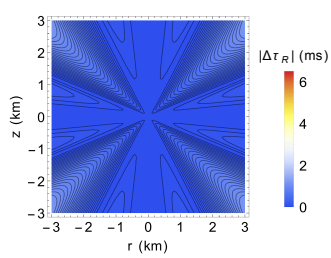

(b)

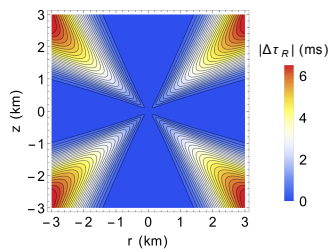

(d)
Figure 3: Similar to Figure 1, but along the azimuth of $90^{\circ}$.

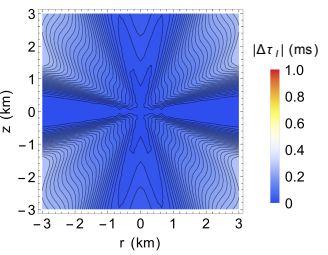

(a)

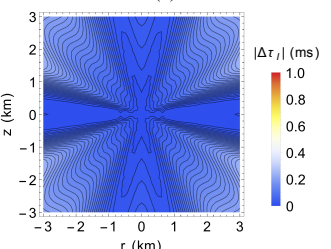

(c)

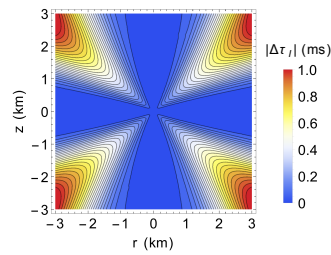

(b)

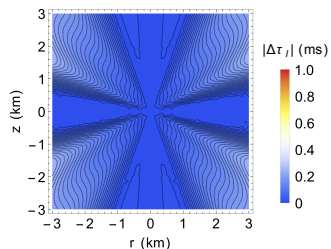

(d)
Figure 4: Similar to Figure 4, but for the imaginary part of the traveltimes.

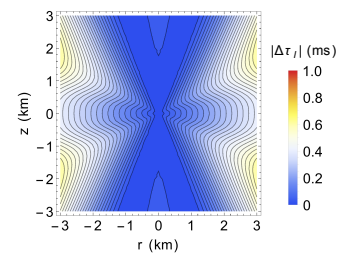

(a)

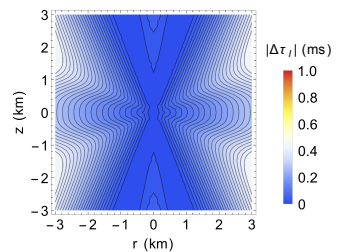

(c)

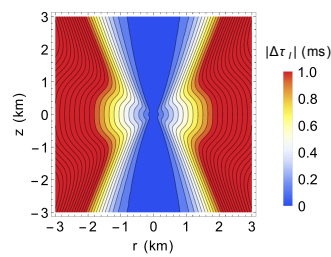

(b)

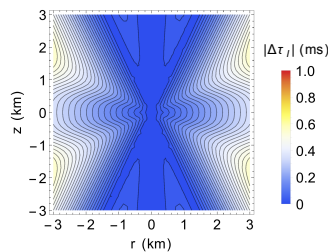

(d)
Figure 5: Similar to Figure 5, but for the imaginary part of the traveltimes. 


\section{Complex traveltimes in attenuating orthorhombic media}

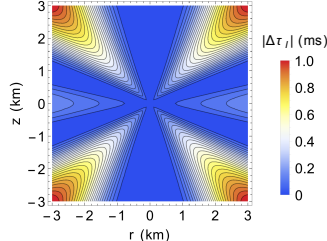

(a)

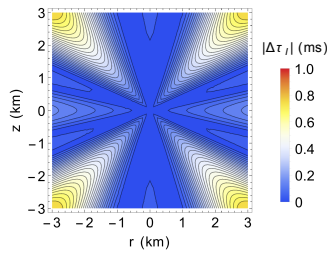

(c)

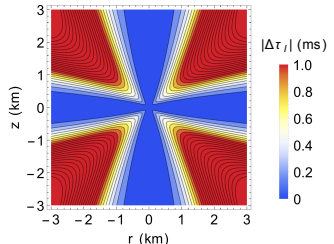

(b)

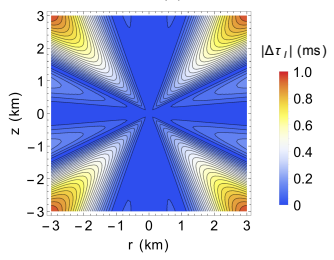

(d)
Figure 6: Similar to Figure 6, but for the imaginary part of the traveltimes.

Considering the values of the Thomsen-style anisotropy parameters in the model, Figure 7 implies that the fast marching scheme is accurate for the orthorhombic models with moderate anelliptical strength in velocity and with strong attenuation anisotropy.

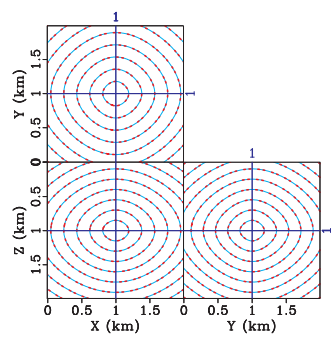

(a)

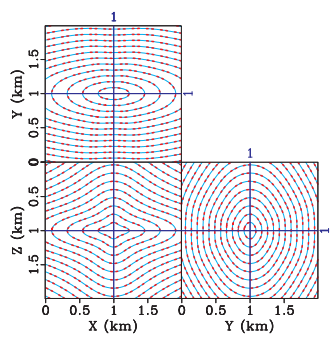

(b)
Figure 7: The contours of the (a) real and (b) imaginary parts of the traveltimes in a homogeneous attenuating orthorhombic model. The model parameters are the same as in the first numerical example. The contour intervals are $50 \mathrm{~ms}$ and $1 \mathrm{~ms}$ for the real and imaginary parts of the traveltimes, respectively. The blue lines correspond to the Shanks solution 2 (equation 16) with the explicit traveltime coefficients. The red dashed lines correspond to the fast marching scheme.

In the third example, we investigate the influence of the attenuation anisotropy parameters on the traveltimes in an inhomogeneous attenuating orthorhombic medium. We design an attenuating orthorhombic version of the French model (French, 1974). A smooth medium model is needed for the fast marching scheme, otherwise the scheme will provide artifacts in the imaginary part of the traveltimes. The first layer in the model is attenuating and isotropic and includes the parameters $v_{P 0}=$ $2.0 \mathrm{~km} / \mathrm{s}$ and $A_{P 0}=0.015$ (corresponding to $Q_{33}=33.3$ ). The second layer is attenuating and orthorhombic and includes the parameters $v_{P 0}=3.2 \mathrm{~km} / \mathrm{s}, v_{x}=3.431 \mathrm{~km} / \mathrm{s}, v_{y}=3.649 \mathrm{~km} / \mathrm{s}$, $\eta_{1}=0.15, \eta_{2}=0.20, \eta_{3}=0.10, A_{P 0}=0.025$ (corresponding to $\left.Q_{33}=20.0\right), \varepsilon_{Q 1}=0.15, \delta_{Q 1}=0.30, \varepsilon_{Q 2}=0.05, \delta_{Q 1}=$ $0.20, \delta_{Q 3}=0.10$. The model is smoothed by a multi-dimensional triangle smoothing program (sfsmooth) from the Madagascar software package (www.ahay.org). Figure 8 compares the traveltimes in the attenuating orthorhombic model and that in the corresponding attenuating model with isotropic attenuation coefficients. The real part of the traveltimes is extremely insensitive to the attenuation anisotropy parameters (Figure 8a), hence it is infeasible to estimate these parameters using the real part of the traveltimes. In contrast, the attenuation anisotropy parameters affect the imaginary part of the traveltimes along an off-vertical direction (Figure 8b), which implies that ignoring the effect of these parameters could weaken the quality of seismic images for steep reflectors in an attenuating orthorhombic media with moderate or strong attenuation anisotropy.

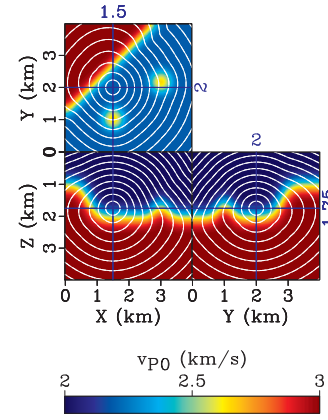

(a)

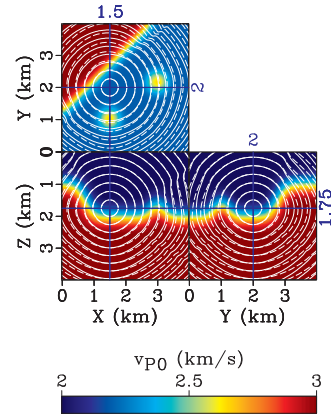

(b)
Figure 8: The contours of the (a) real and (b) imaginary parts of the traveltimes in the attenuating orthorhombic French model. The white solid lines correspond to the attenuating orthorhombic model. The dashed lines correspond to the similar model but with isotropic attenuation coefficients $\left(\varepsilon_{Q 1}=\right.$ $\varepsilon_{Q 2}=\delta_{Q 1}=\delta_{Q 2}=\delta_{Q 3}=0$ ). The background behind the contours shows the $v_{P 0}$ model. The point source is located at $x=1.5 \mathrm{~km}, y=2.0 \mathrm{~km}$ and $z=1.75 \mathrm{~km}$. The contour intervals in the plots (a) and (b) are $0.1 \mathrm{~s}$ and $2 \mathrm{~ms}$, respectively. In the plot (a), the white dashed lines are overlaid with the solid white lines.

\section{CONCLUSIONS}

We developed a numerical scheme to calculate attenuation in an acoustic orthorhombic medium using a complex traveltime eikonal formulation. The solution is based on series-based approximations of the sixth order eikonal equation, with Shanks transform to improve the accuracy of the series. Shanks transform solution 2 formulated with respect to the perturbation parameters scaling the anellipticity parameters proved to be the most accurate among all of the proposed solutions. Such a solution was incorporated into a fast marching scheme to calculate complex traveltimes in inhomogeneous attenuating orthorhombic models with moderate anellipticity and strong attenuation. The inhomogeneity, however, is required to be smooth to avoid artifacts in the traveltimes. We are developing more accurate and stable methods applicable to strongly inhomogeneous, attenuating orthorhombic media that we might share in the presentation of the work at the meeting. 


\section{REFERENCES}

Alkhalifah, T., 2000, An acoustic wave equation for anisotropic media: Geophysics, 65, 1239-1250, https://doi.org/10.1190/1.1444815.

Alkhalifah, T., 2011, Scanning anisotropy parameters in complex media: Geophysics, 76, no. 2, U13-U22, https://doi.org/10.1190/1.3553015.

French, W. S., 1974, Two-dimensional and three-dimensional migration of model-experiment reflection profiles: Geophysics, 39, 265-277, https://doi org/10.1190/1.1440426.

Hao, Q., and T. Alkhalifah, 2017a, An acoustic eikonal equation for attenuating orthorhombic media: Geophysics, 82, no. 4, WA67-WA81, https://doi .org/10.1190/geo2016-0632.1.

Hao, Q., and T. Alkhalifah, 2017b, An acoustic eikonal equation for attenuating transversely isotropic media with a vertical symmetry axis: Geophysics, 82, no. 1, C9-C20, https://doi.org/10.1190/geo2016-0160.1.

Hao, Q., and T. Alkhalifah, 2017c, Acoustic eikonal solutions for attenuating VTI media: 87th Annual International Meeting, SEG, Expanded Abstracts, 289-293, https://doi.org/10.1190/segam2017-17661832.1.

Vavrycuk, V., 2006, Calculation of the slowness vector from the ray vector in anisotropic media: Proceedings of the Royal Society, 462, 883-896, https://doi.org/10.1098/rspa.2005.1605.

Vavrycuk, V., 2007, Ray velocity and ray attenuation in homogeneous anisotropic viscoelastic media: Geophysics, 72, no. 6, D119-D127, https://doi .org/10.1190/1.2768402.

Zhu, Y., and I. Tsvankin, 2007, Plane-wave attenuation anisotropy in orthorhombic media: Geophysics, 72, no. 1, D9-D19, https://doi.org/10.1190/1 .2387137 . 